\title{
Predicting the Collapse of Turbulence in Stably Stratified Boundary Layers
}

\author{
B. J. H. van de Wiel • A. F. Moene • G. J. Steeneveld • \\ O. K. Hartogensis • A. A. M. Holtslag
}

Received: 26 June 2006 / Accepted: 30 October 2006 /

Published online: 28 July 2007

(C) Springer Science + Business Media B.V. 2007

\begin{abstract}
The collapse of turbulence in a plane channel flow is studied, as a simple analogy of stably stratified atmospheric flow. Turbulence is parameterized by first-order closure and the surface heat flux is prescribed, together with the wind speed and temperature at the model top. To study the collapse phenomenon both numerical simulations and linear stability analysis are used. The stability analysis is nonclassical in a sense that the stability of a parameterized set of equations of a turbulent flow is analyzed instead of a particular laminar flow solution. The analytical theory predicts a collapse of turbulence when a certain critical value of the stability parameter $\delta / L$ (typically $\mathrm{O}(0.5-1)$ ) is exceeded, with $\delta$ the depth of the channel and $L$ the Obukhov length. The exact critical value depends on channel roughness to depth ratio $z_{0} / \delta$. The analytical predictions are validated by the numerical simulations, and good agreement is found. As such, for the flow configuration considered, the present framework provides both a tool and a physical explanation for the collapse phenomenon.
\end{abstract}

Keywords Relaminarization $\cdot$ Stable boundary layer $\cdot$ Hydrodynamic stability

\section{Introduction}

It is now over 20 years ago that Frans Nieuwstadt formulated his elegant framework on the turbulent structure of the stably stratified, nocturnal boundary layer [28]. The original study departs from a second order closure model with parameterized equations for variances and covariances. The turbulence model is further simplified by assuming that both the tendency and the transport terms can be neglected in stably stratified conditions, thus assuming local equilibrium of TKE. Under these assumptions, it was shown that all dimensionless turbulent quantities can be expressed as a function of a

B. J. H. van de Wiel $(\bowtie) \cdot$ A. F. Moene • G. J. Steeneveld • O. K. Hartogensis • A. A. M. Holtslag Department of Meteorology and Air Quality, Wageningen University and Research Centre, Wageningen, The Netherlands e-mail: Bas.vandeWiel@wur.nl 
single dimensionless parameter $z / \Lambda . \Lambda$ is the so-called local Obukhov length scale, which is based on the local fluxes of momentum and heat. As such this local similarity theory can be seen as a generalization of the traditional [27] Monin-Obukhov similarity that is based on surface fluxes. Since its introduction by Nieuwstadt, the local similarity theory has found a lot of observational support, so that nowadays the framework seems to be well-established in boundary-layer theory $[5,7,11]$.

In the same paper Nieuwstadt (from now on N84) develops a simple equilibrium model for the stable boundary layer (SBL) as a whole, in order to construct profiles for the mean wind and temperature and for the turbulent fluxes. To this end, the Reynoldsaveraged equations for the mean variables are solved by the closure assumption that $R_{f}$ and $R_{i}$ are equal to their critical value in the whole SBL. The latter result is in agreement with local scaling in the limit $z / \Lambda \rightarrow \infty$, that is in the so-called $z$-less limit (see also: Wyngaard, [42] and Holtslag and Nieuwstadt, [15]). Although, according to the authors, this closure assumption is rather restricted (and will be relaxed in the present study), the equilibrium profiles that are calculated are generally reasonably realistic in the bulk of the boundary layer as compared to observational data (e.g. N84). At this point it is important to note that the aforementioned agreement is only observed for cases with moderate to large mechanical forcing (geostrophic wind speed $>5 \mathrm{~m} \mathrm{~s}^{-1}$ ), were the boundary layer is said to be weakly stable (WSBL). Also, as a direct consequence of the closure assumption, the profiles are rather unrealistic near the surface. This latter shortcoming however, can be corrected (e.g. [5, 29]) or may be circumvented by adopting less stringent closure assumption (present study). Thus, in summary, the N84 theory provides us a solid physical picture of the weakly stratified SBL.

In contrast, observed stable boundary layers with low mechanical forcing (say geostrophic wind speed $<5 \mathrm{~m} \mathrm{~s}^{-1}$ ), do not seem to indicate quasi-steady behaviour as in N84 [41]. Typically under these circumstances turbulence is not able to maintain its continuous character. This means that the turbulence collapses to extremely low intensities and as a consequence the large scale atmospheric flow becomes more or less 'decoupled' from the surface [8, 24]. Then, after some time, turbulent activity may suddenly increase again, followed by another collapse. Such a sequence of events is sometimes referred to as 'global intermittency', in a sense that in the periods of weak turbulence eddies on all scales are missing or suppressed [22]. Apparently, at this stage the SBL has entered different regime, often referred to as the very stable boundary layer regime (VSBL). Intuitively, the transition between both regimes can be anticipated based on simple gradient-transfer thinking: in case of weak stratification a sudden increase in the vertical temperature gradient generally causes a larger downward heat flux, which opposes the gradient disturbance (negative feedback). In case of strong stratification however, a sudden increase in the vertical temperature gradient causes a significant decrease of the turbulent diffusion coefficient, which may lead to a smaller flux so that the disturbance is enhanced (positive feedback).

In the present work we investigate whether it is possible to predict the limit where the SBL gives up its steady behaviour. In other words: the aim is to find the point where turbulence collapses and were the WSBL changes regime towards the VSBL.

The rationale behind our method is best explained by a quote originating from Landau and Lifschitz as cited from Drazin and Reid [9]:

Yet not every solution of the equations of motion, even if it is exact, can actually occur in nature. The flows that occur in nature must not only obey the equations of fluid dynamics, but must also be stable. 
In the present work this is interpreted as follows: we hypothesize that:

(1) The continuous turbulent solutions of the SBL are (hydrodynamically) stable for high mechanical forcing, and are therefore observed in nature.

(2) The continuous turbulent solutions of the SBL are (hydrodynamically) unstable for low mechanical forcing, and are therefore not observed in nature. In this second case a collapsed SBL will be observed.

The statement above summarizes the core of hydrodynamic stability theory, which has a long proven history in fluid dynamics (e.g. [3, 9]). However its potential related to the problem above, i.e. the prediction of the turbulent collapse in the SBL, seems not to be fully recognized so far. In a classical sense, analytical stability analysis in stably stratified flows has often been applied by studying the behaviour of perturbations of fully known solutions of the equations of motion [30]. In the case of the frictionless Boussinesq equations, the perturbations are governed by the well-known Taylor-Goldstein equation. From this equation, Miles [26] and Howard [16] showed that a necessary condition for hydrodynamic instability of a linearly stratified flow is $R i<1 / 4$ (Note that, as in the present study, the theoretical studies above assume exponential growth/decay of disturbances, excluding possible algebraic growth/decay as e.g. in Hanazaki and Hunt [12]). As such, this Ri-criterion may serve as a tool to predict the transition from a stably stratified laminar flow to a turbulent flow. It is tempting to apply the same theory of hydrodynamic stability to the reverse problem, i.e. by predicting the collapse of a turbulent stratified flow towards a laminar state. However, it is clear that this is not straightforward, because closed-form analytical solutions of the turbulent reference state are generally unavailable. Again, an analytical formulation of the reference state is necessary in order to impose the perturbation analysis on it.

In order to tackle this problem, an innovative approach was given by Derbyshire [6-8]. In absence of time-dependent exact solutions of the equations of motion in a turbulent reference state, Derbyshire studied the hydrodynamic stability of a parameterized form of the Reynolds-Averaged Navier-Stokes equations (RANS) for a stably stratified flow. First, Derbyshire (from now on D99) uses numerical simulations with RANS (first-order closure) to show that, in essence, the collapse of turbulence is a real physical phenomenon that arises from a positive feedback between the turbulent transport in the SBL and the surface.

Second, D99 attempted to generalize these numerical results by performing an analytical linear stability analysis on linear wind and temperature profiles. The results of this analysis are promising in a qualitative sense, namely that some profiles become unstable to perturbations for increasing stratification, which agrees with the numerical findings in the same study. However, the theoretical assumption of linearity of the profiles prohibits a direct quantitative comparison with the numerical model, which does not need this restriction. Moreover, this assumption, albeit mathematically attractive, is physically unrealistic close to the surface. This is because profiles tend to be logarithmic rather than linear close to the surface. This, in turn, is a direct consequence of the fact that in this region the size of the dominant turbulent eddies scales with height.

Therefore, the aim of the present work is to extend the stability analysis of D99 to the more general case with realistic profiles. Such analysis enables future comparison of analytical results with observations, so that predictions of turbulence collapse in the SBL can be made.

Finally, we mention an interesting perspective to the instability problem by McNider et al. [25] and later by Van de Wiel et al. [39, 40]. These studies simplify the SBL equations to a truncated system of a few coupled nonlinear differential equations. By using a system 
dynamics approach they obtained intriguing results, showing: instability, oscillations, bifurcations and potential loss of predictability. Although the models used are too simplified to be more than a suggestive of SBL behaviour, they may support an alternative picture to the N84 work. In the present work our results will also be interpreted from a system dynamics point of view.

The paper is organized as follows: in Section 2 a simple model is formulated and main results of the numerical simulations are given. Next, the set of model equations is analyzed analytically leading to the equilibrium solutions in Section 3. Also the stability of the equilibrium is analyzed (Section 3), which leads to an expression for the marginal state that marks the transition from stability to instability of the flow. The analytical predictions are verified by comparison with the numerical outcome in Section 4. Finally, a discussion of the results is given in Section 5, followed by the conclusions.

\section{A Simple Model of Stratified Flow}

\subsection{Model set up}

As an analogy to atmospheric flow, a 1-dimensional model of stratified flow is set up. The model set up is such that in its stationary state the temperature and wind profiles follow classical Monin-Obukhov similarity theory [27] which means that they are very similar to reported observational profiles in the atmospheric surface layer.

Some characteristics of the model are (Fig. 1, common notation):

- The height of the model $\delta$ is fixed.

- Both the wind speed and the temperature at the top of the model are prescribed: $U_{\text {TOP, }} T_{\text {TOP. }}$

- At the surface the heat flux $H_{0}$ is prescribed and a no slip condition for wind is applied by assuming a roughness length for momentum $z_{0}=0.1[\mathrm{~m}]$.

- Coriolis effects are ignored.

- Long wave radiative divergence is not taken into account.

- Turbulent transport is modeled by using first order (local) closure (gradienttransfer) based on the gradient Richardson number Ri. This closure assumes that turbulent transport terms are small under stably stratified conditions and that the shear production, the buoyancy destruction and the dissipation of TKE are in local equilibrium. A large number of studies provide observational support to the local

Fig. 1 Schematic picture of the channel flow system

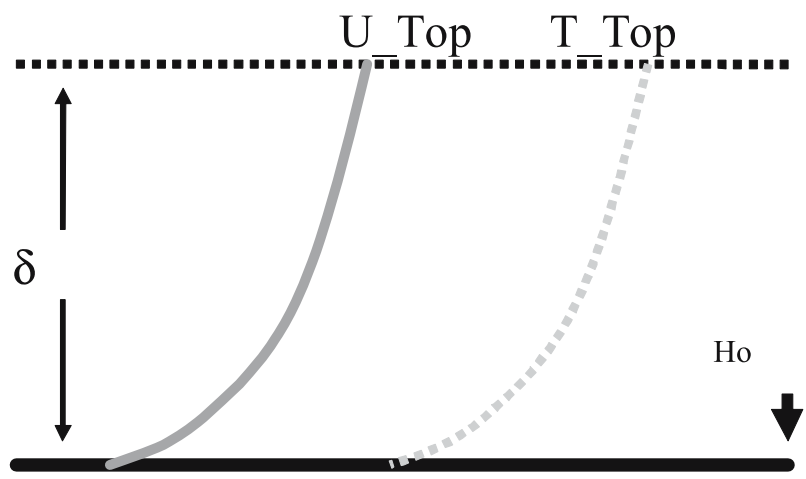


equilibrium assumption in stably stratified boundary layers (e.g. [10, 19, 28, Basu et al. 2006, 37]). The robustness of the local equilibrium assumption may not be surprising in view of the fact that vertical mixing is limited by stratification, so that large transport of TKE as in the convective BL is not likely.

Our governing equations for wind speed $U$ and temperature $T$ read :

$$
\begin{gathered}
\frac{\partial U}{\partial t}=\frac{1}{\rho} \frac{\partial \tau}{\partial z} \\
\frac{\partial T}{\partial t}=-\frac{1}{\rho c_{\mathrm{p}}} \frac{\partial H}{\partial z}
\end{gathered}
$$

The turbulent stress $\tau$ and the turbulent heat flux $H$ are parametized via (local closure):

$$
\frac{\tau}{\rho}=K_{m} \frac{\partial U}{\partial z} \text { and } \frac{H}{\rho c_{\mathrm{p}}}=-K_{H} \frac{\partial T}{\partial z}
$$

Here, the turbulent diffusivity is given by:

$$
K_{H, m}=l_{n}^{2}(\partial U / \partial z) f(\mathrm{Ri})
$$

with the neutral mixing length $l_{n}=\kappa z$ and

$$
\begin{aligned}
& f(\mathrm{Ri})=\left(1-\frac{\mathrm{Ri}}{R_{\mathrm{c}}}\right)^{2} ; \quad \mathrm{Ri} \leq R_{c} \\
& f(\mathrm{Ri})=0 ; \quad \mathrm{Ri}>R_{\mathrm{c}}
\end{aligned}
$$

The gradient Richardson number is defined here as:

$$
\mathrm{Ri} \equiv \frac{\mathrm{g}}{\mathrm{T}_{\mathrm{ref}}} \frac{\partial \mathrm{T} / \partial \mathrm{z}}{(\partial \mathrm{U} / \partial \mathrm{z})^{2}}
$$

The Von Kármán constant $\kappa$ is taken to be 0.4 [14] and both the density $\rho$ of the air $(1.2$ $\left.\left[\mathrm{kg} \mathrm{m}^{-3}\right]\right)$ and the specific heat capacity at constant pressure $c_{\mathrm{p}}\left(1005\left[\mathrm{~J} \mathrm{~kg}^{-1} \mathrm{~K}^{-1}\right]\right.$ are assumed to be constant. Furthermore, the reference temperature $T_{\text {ref }}$ of the system is fixed at $285[\mathrm{~K}]$ and $g=9.81\left[\mathrm{~m} \mathrm{~s}^{-2}\right]$.

The 'critical' Richardson number $R_{\mathrm{c}}$ is set to 0.2 . It is important to notice that the impact of this formulation is not trivial in a sense that the collapse of turbulence occurs whenever $R_{\mathrm{c}}$ is exceeded. On the contrary, it will be shown (Section 5) that the collapse of turbulence always sets in before this critical value is reached anywhere in the channel. Thus the effect of the critical Richardson-formulation is only indirect, in a way that it influences the dependence of the turbulent eddy-diffusivity $K_{H, m}$ on Ri. Our $R_{\mathrm{c}}$ of 0.2 and the shape of the stability function $f(\mathrm{Ri})$, are chosen such that they are in accordance with the observed loglinear type of local-scaling, which is nowadays widely accepted up to a stability range of $z / L=O(1)$ (e.g. Businger et al. [2], Högström, [14] and references therein). Thus, from a physical point of view, the formulations above include the limiting effects on dominant eddy size of both stability and the presence of a solid boundary. 
The log linear character of the wind and temperature profiles requires a very fine numerical discretization near the surface in order to obtain accurate results. The results need to be accurate in order to be comparable with the analytical analysis. Therefore, we use a grid spacing $\Delta z$ of $0.2[\mathrm{~m}]$ near the surface with a stretch factor of $5 \%$ upwards. With 40 layers the resulting model domain $\delta$ becomes $23.6[\mathrm{~m}]$. The equations are integrated in time using a fourth order Runge Kutta scheme with a time step of 0.1 [s] to ensure numerical stability.

\subsection{Numerical simulation: results}

In order to investigate the collapse phenomenon, the response of the system to increased cooling is examined. To this end, the wind speed at the top is fixed at $4\left[\mathrm{~ms}^{-1}\right]$ and four different simulations are done for different fixed surface heat flux values $H_{0}$. Alternatively we could have varied $U_{\text {TOP }}$ between the cases and keep a single value for $H_{0}$, which would lead to similar results. The four simulations start from a neutral state, so that the simulations have the same initial friction velocity $u_{*}$. Figure 2 shows the modeled time evolution of the friction velocity for the cases as an indicator of the evolution of turbulence intensity in response to different cooling rates.

We observe that a stationary situation is reached for $H_{0}=-10$ and $-15.25\left[\mathrm{~W} \mathrm{~m}^{-2}\right]$, the latter being the limiting case of the stationary solutions for the fixed $U_{\text {TOP }}$ value indicated above. The limiting case corresponds to $\delta / L=0.52$. The models response to a slightly larger cooling rate of $-15.40\left[\mathrm{~W} \mathrm{~m}^{-2}\right]$ is not smooth as might have been expected. Instead, a dramatic change of the results is observed, with the friction velocity rapidly going to zero, indicating a total collapse of turbulent intensity. Apparently, the flow is unable to maintain a stationary turbulent state with this prescribed cooling rate at the surface. For completeness in Fig. 3 the equilibrium values of $u *$ for a whole family of runs with different $H_{0}$ is shown. The numerical integration period was set to $5 \mathrm{~h}$, which turned out to be sufficient to reach reasonable stationarity. In accordance with the results above, the line of equilibrium states behaves smoothly until a certain maximum value of $H_{0}$ is exceeded.

In the next section we look into more detail at the limiting continuous turbulent case $H_{0}=-15.25\left[\mathrm{~W} \mathrm{~m}^{-2}\right]$ and at the collapsed case $H_{0}=-15.40\left[\mathrm{~W} \mathrm{~m}^{-2}\right]$, in order to gain more insight in the model results.

Fig. 2 Modeled time evolution of the friction velocity for four different cooling rates. *The equilibrium values for $\delta / L$ are given except for the collapsed cases where no turbulent equilibrium is reached

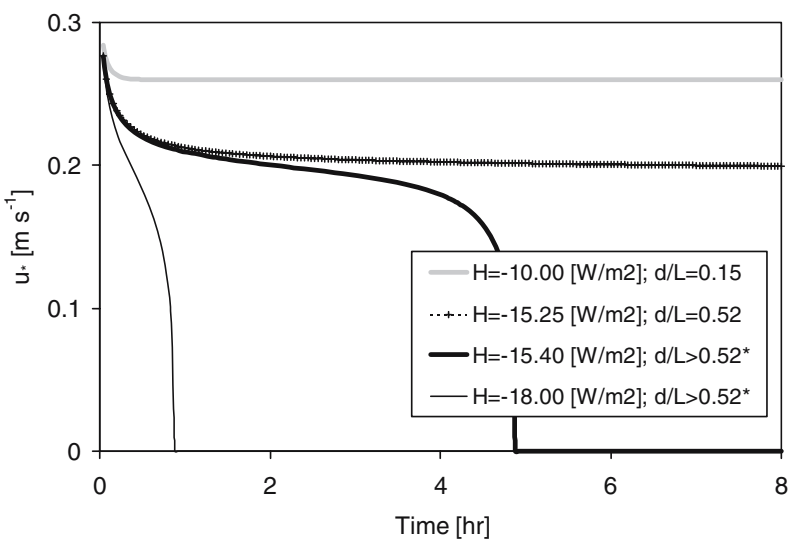




\subsubsection{The continuous turbulent case}

Figure $4 \mathrm{a}$ and $\mathrm{b}$ depict the evolution of the wind and temperature profiles for the continuous turbulent case. Both wind and temperature profiles evolve from a neutral initial state towards a stably stratified steady state. Although the theoretical analysis is discussed in Section 3, we anticipate the results of this section, by comparing the numerical equilibrium profiles with the profiles obtained by the analytical equilibrium solutions. Profiles after $10 \mathrm{~h}$ of integration are shown to ensure stationarity. Indeed, a good correspondence between the numerical and the analytical results is found.

\subsubsection{The collapse case}

In Fig. 5a and $\mathrm{b}$ the evolution of the wind and temperature profiles for the collapse case is given. Figure 5a shows that the wind profile changes from a logarithmic shape to a more log-linear shape at large stability. Consequently, during the process of collapse, the wind close to the surface becomes weaker than initially, during the neutral conditions. The effect of the collapse of turbulence is also evident in Fig. 5b. After a gradual initial cooling of $2 \mathrm{~K}$ in about $4.5 \mathrm{~h}$, the boundary layer starts to cool very rapidly (over $1 \mathrm{~K}$ in $20 \mathrm{~min}$ ). During the collapse process we observed a rapid decrease of the turbulent stress over the whole layer and a strong divergence of the turbulent heat flux across the layer (not shown).

The process in Fig. 4b is sometimes referred to as 'runaway-cooling' [20]. In numerical weather prediction this process may lead to continuation problems, and often, for practical reasons, this process is suppressed by an artificial modification of the eddy diffusion coefficients [20]. However, this 'runaway-cooling' is a realistic physical feature of the SBL [41]. At the same time, atmospheric observations show that the runaway behaviour of the surface cooling 'stops' after a few hours, with typical temperature differences between surface and SBL-top temperatures of $\mathrm{O}(5-10)[\mathrm{K}]$. This natural limitation to cooling is the result of strong negative feedbacks in the net longwave radiation and in the soil heat flux. This means that eventually a temperature equilibrium in absence of a turbulent heat flux is reached [39-41]. The present model configuration does not take into account these feedbacks, which explains the rather extreme response of the temperature stratification to the collapse of turbulence in our model.

Fig. 3 Equilibrium values of $u_{*}$ (after $5 \mathrm{~h}$ of integration) as a function of imposed surface heat flux

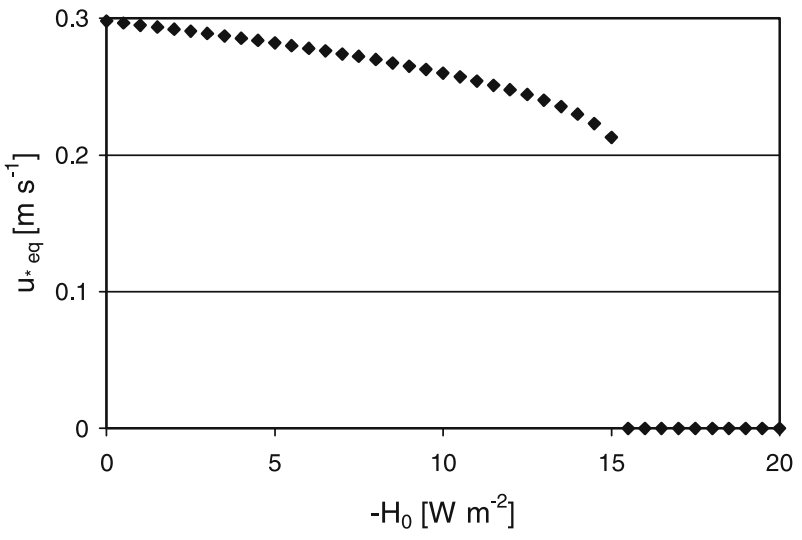




\section{Analytical Approach}

3.1 The equilibrium solution

In our system the governing equations for the conservation of momentum and heat are:

$$
\begin{gathered}
\frac{\partial U}{\partial t}=\frac{1}{\rho} \frac{\partial \tau}{\partial z} \\
\frac{\partial T}{\partial t}=-\frac{1}{\rho c_{\mathrm{p}}} \frac{\partial H}{\partial z}
\end{gathered}
$$

Fig. 4 a Modeled evolution of the wind profile for the 'continuous turbulent' case. Also the analytical solution for the steady state is plot according to Eq. 12 . b As in (a) but for temperature and Eq. 13

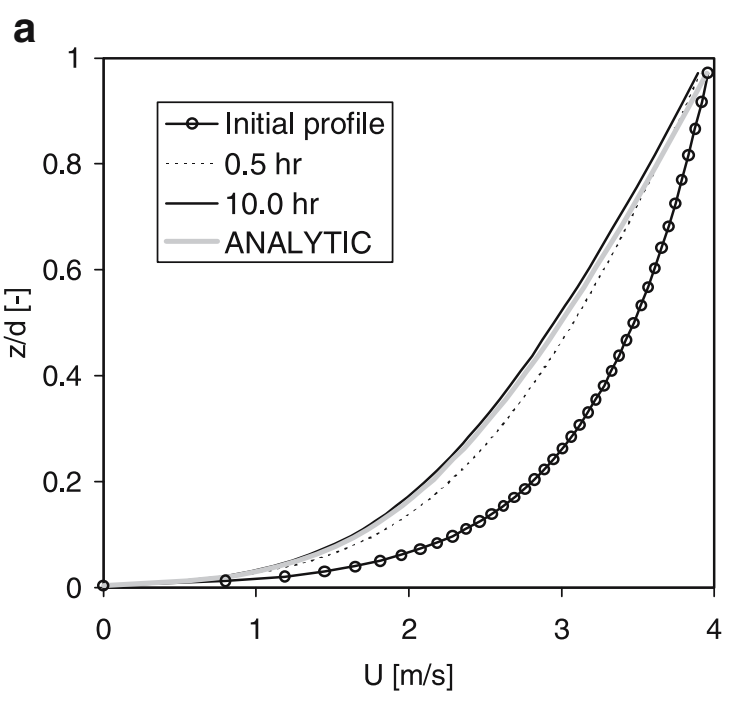

b

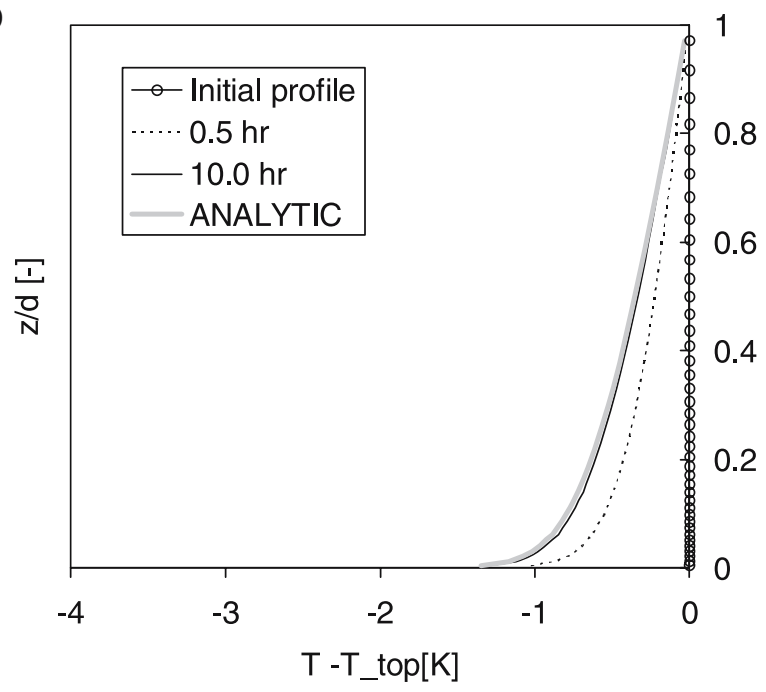


Under the given closure assumptions (Eq. 3), this becomes:

$$
\begin{gathered}
\frac{\partial U}{\partial t}=\frac{\partial}{\partial z}\left((\kappa z)^{2}(\partial U / \partial z)^{2} f(\mathrm{Ri})\right) \\
\frac{\partial T}{\partial t}=\frac{\partial}{\partial z}\left((\kappa z)^{2}(\partial T / \partial z)(\partial U / \partial z) f(\mathrm{Ri})\right)
\end{gathered}
$$

Fig. 5 a Evolution of the wind profile for the 'decoupled' case. $\mathbf{b}$ As in (a) but for temperature
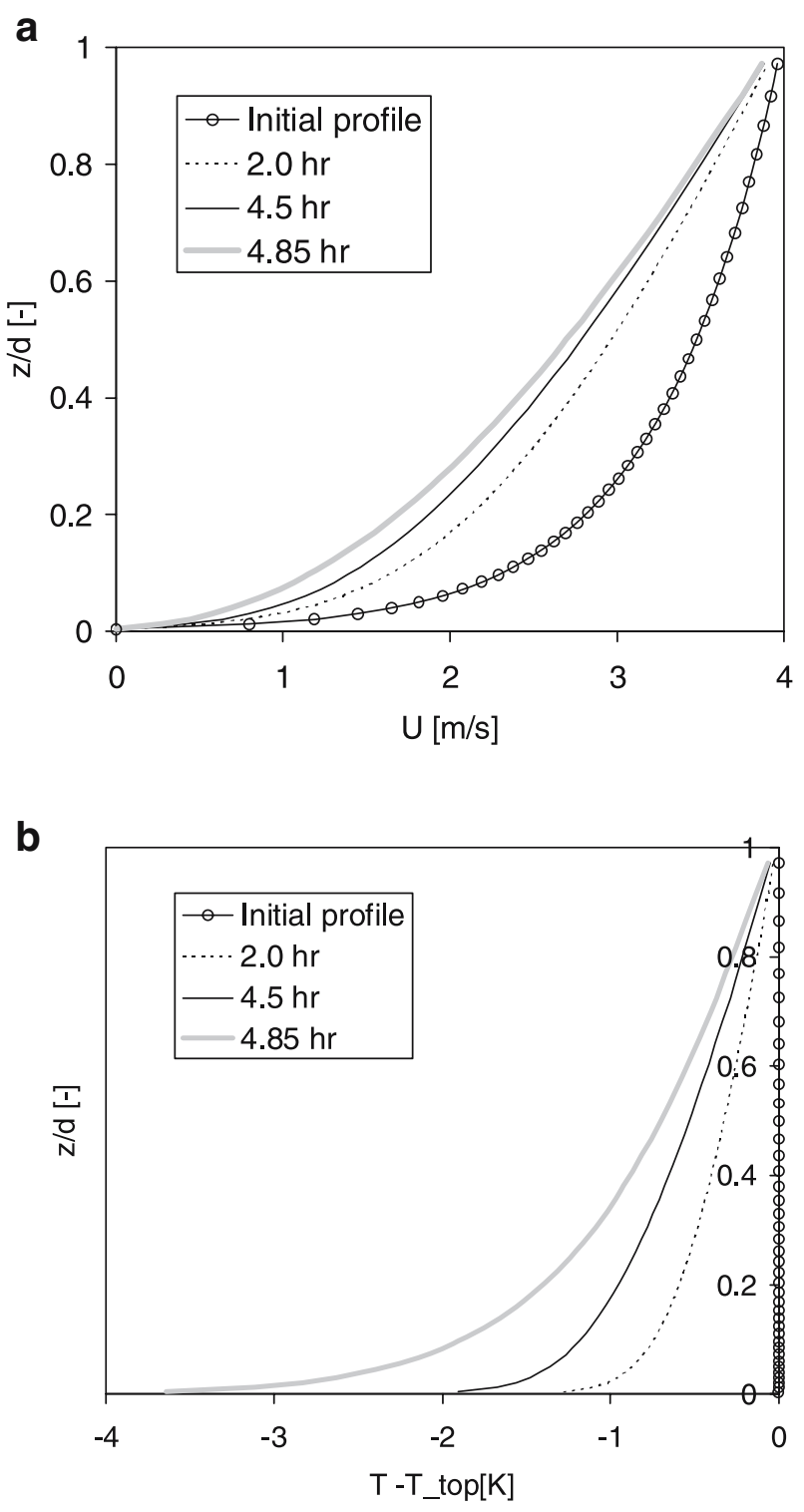
The equilibrium profiles of wind $U(z)_{\mathrm{eq}}$ and temperature $T(z)_{\mathrm{eq}}$ are found by putting the 1.h.s. of the Eqs. 5 and 6 to zero. Then, the equilibrium fluxes are constant with height:

$$
\tau / \rho=\tau_{0} / \rho=C_{1}^{2} \equiv u_{*}^{2} \text { and }-H /\left(\rho c_{\mathrm{p}}\right)=-H_{0} /\left(\rho c_{\mathrm{p}}\right)=C_{1} C_{2} \equiv u_{*} \theta_{*}
$$

(Note that, for convenience, the constants are defined such that: $C_{1}=u *$, and $C_{2}=\theta *$ ). After some algebraic manipulation (Appendix A), log-linear shapes for $U(z)_{\mathrm{eq}}$ and $T(z)_{\mathrm{eq}}$ are found:

$$
\begin{aligned}
& U(z)_{\mathrm{eq}}=\frac{u_{*}}{\kappa}\left(\ln (z)+\alpha \frac{z}{L}\right)+C_{3} \\
& T(z)_{\mathrm{eq}}=\frac{\theta_{*}}{\kappa}\left(\ln (z)+\alpha \frac{z}{L}\right)+C_{4}
\end{aligned}
$$

with: $L \equiv \frac{u_{*}^{2}}{\theta_{*}} \frac{T_{\mathrm{ref}}}{\kappa g}$, the classical Obukhov length and $\alpha \equiv 1 / R_{\mathrm{c}}=5$.

The solution has four unknowns $\left(u *, \theta *, C_{3}, C_{4}\right)$ and requires four boundary conditions:

$$
\begin{gathered}
U\left(z=z_{0}\right)=0 \\
H\left(z=z_{0}\right)=H_{0} \\
U(z=\delta)=U_{\mathrm{TOP}} \\
T(z=\delta)=T_{\mathrm{TOP}}
\end{gathered}
$$

Condition (I) and (II) are inserted in Eq. 9 to eliminate $C_{3}$ and $\theta_{*}$ (in $L$ ). Then we impose (III) to leave a single equation for $u_{*}$. Some rearrangement gives:

$$
\widehat{u}_{*}^{3}-\widehat{u}_{*}^{2}-\widehat{H}=0
$$

with: $\widehat{u}_{*}=u_{*} / u_{* N}$ and

$$
u_{* N}=\frac{\kappa U_{\mathrm{TOP}}}{\ln \left(\delta / z_{0}\right)} ; \widehat{H}=\frac{H_{0}}{u_{* N}^{3}} \cdot \frac{\alpha \kappa g}{\rho c_{p} \theta_{\mathrm{ref}}} \frac{\left(\delta-z_{0}\right)}{\ln \left(\delta / z_{0}\right)}
$$


From Eq. $11 u_{*}$ is found and $\theta_{*}$ is found directly by the definition $\theta_{*}=-H_{0} /\left(\rho c_{\mathrm{p}} u_{*}\right)$.

Inserting $\mathrm{BC}(\mathrm{IV})$ finally leads to the equilibrium profiles of our system:

$$
\begin{gathered}
\frac{U(z)_{\mathrm{eq}}}{u_{* \mathrm{eq}}}=\frac{1}{\kappa}\left(\ln \left(\frac{z}{z_{0}}\right)+\alpha \frac{\left(z-z_{0}\right)}{L_{\mathrm{eq}}}\right) \\
\frac{T_{\mathrm{TOP}}-T(z)_{\mathrm{eq}}}{\theta_{* \mathrm{eq}}}=\frac{1}{\kappa}\left(\ln \left(\frac{\delta}{z}\right)+\alpha \frac{(\delta-z)}{L_{\mathrm{eq}}}\right)
\end{gathered}
$$

Note that Eqs. 12 and 13 are the well-known log-linear profiles that follow MoninObukhov theory (Section 2). In fact, this particular result could be anticipated, in a sense that a constant flux layer inevitably leads to this classical form. Nevertheless, the formal derivation is given, to facilitate future application to other flow configurations (such as pressure driven flow), that may have different boundary conditions. In Section 2 is was shown that the solutions given by Eqs. 12 and 13 agree with the numerical equilibrium profiles (Fig. 4a,b).

\subsection{An equilibrium diagram}

The results of the previous section are now summarized by means of an equilibrium diagram that plots the whole collection of equilibrium points according to (11) (Fig. 6). In this diagram a scaled model result is plot against a forcing parameter. For our system $\widehat{H}$ is the forcing parameter and $\widehat{u}_{*}$ the result. Interestingly, Fig. 6 reveals that two real solutions for $\widehat{u}_{* e q}$ are obtained for a given $\widehat{H}$ (the third (unphysical) root being negative and real). The duality of solutions is a direct consequence of the nonlinear dependence of turbulent diffusion on the vertical temperature gradient (Section 1) and has been recognized in meteorology for some time (Taylor [38]; De Bruin [4]; Malhi [21]). For the specific configuration discussed in the present work, Taylor [38] conjectured that one of the two solutions above is expected to be unstable, although a formal prove for this statement is not given. In fact our numerical results of Section 2 also indicate only one solution branch at a

Fig. 6 Equilibrium values of $\widehat{u} *$ as a function of the imposed surface heat flux $-\widehat{H}$. Dimensionless parameters are plot according to Eq. 11 (note the minus sign in $-\widehat{H}$ to plot positive values)

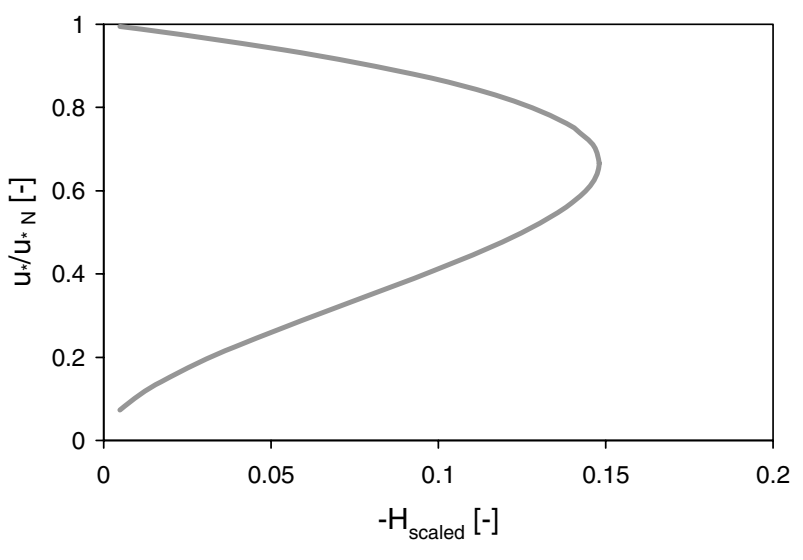


given $\widehat{H}$ in accordance with Taylor's statement. The stability analysis in the present work will provide a formal proof to Taylor's conjecture.

Besides the 'duality-issue', from Fig. 6 another interesting feature comes out. It occurs that beyond a certain maximum cooling $\widehat{H}_{T}$ no equilibrium solution exists. Of course, in unscaled terms this implies either a maximum $H$ at given $U_{\text {TOP }}$ or a minimum $U_{\text {TOP }}$ at a given $H$. The point $\left(\widehat{H}_{T}, \widehat{u}_{* T}\right)$ is called a 'limiting' or 'turning' point of the solution. For our system the turning point has the numerical value $\left(-\widehat{H}_{T}, \widehat{u}_{* T}\right)=\left(\frac{4}{27}, \frac{2}{3}\right)$. From this the expression for the dimensional maximum heat flux reads:

$$
|H|_{\max }=\left|-\frac{4}{27} u_{* N}^{3} \frac{1}{\alpha} \frac{\rho c_{\mathrm{p}} \theta_{\text {ref }}}{\kappa g} \frac{\ln \left(\delta / z_{0}\right)}{\left(\delta-z_{0}\right)}\right|\left[W m^{-2}\right]
$$

The physical background of this maximum is readily understood from the fact that it is proportional to the cube of a friction velocity scale (power) multiplied by the critical Richardson number (i.e. 1/ $\alpha$ ) and some numerical constants (compare also: Derbyshire [5]).

Finally, we evaluate the stability parameter $\delta / L$ at the turning point. The condition $\left(-\widehat{H}_{T}, \widehat{u}_{* T}\right)=\left(\frac{4}{27}, \frac{2}{3}\right)$ leads to: $u_{* T}=\frac{2}{3} u_{* N}$ and

$$
\theta_{* T}=\frac{4}{27} \frac{u_{* N}^{3}}{u_{* T}} \frac{1}{\alpha} \frac{\rho c_{\mathrm{p}} \theta_{\mathrm{ref}}}{\kappa g} \frac{\ln \left(\delta / z_{0}\right)}{\left(\delta-z_{0}\right)}
$$

Inserting the result above into the definition of the Obukhov length finally leads to an expression for the stability parameter at the turning point:

$$
\frac{\delta}{L_{T}}=\frac{\ln \left(\frac{\delta}{z_{0}}\right)}{2 \alpha\left(1-\frac{z_{0}}{\delta}\right)}
$$

This result will be considered in the next section.

\subsection{Linear stability analysis}

As stated in the introduction, the behaviour of the system is not only characterized by the equilibrium itself but also by the stability of this equilibrium. Therefore we investigate the stability of the equilibrium solution of the previous section by means of linear stability analysis. As such, the governing equations (Eqs. 1 and 2) are linearized around their equilibrium. To obtain information on the stability of equilibria, usually exponentially growing/decaying perturbations are studied (e.g. [34]), because it is assumed that this type of perturbations is likely to become dominant as time proceeds. However, perturbations that do not grow exponentially may still grow algebraically (see e.g. discussion in Hanazaki and Hunt [12]). Thus, in principle, this particular choice of the perturbation restricts the generality of the results, so that comparison with the numerical simulations remains necessary in order to draw conclusions. In our case, such a verification is presented in 
Section 4. Also, note that we restrict ourselves to 1-dimensional perturbations that depend only on $z$. Thus the scaled form of our perturbations read:

$$
\begin{aligned}
& \widehat{U}_{\mathrm{P}}(z, t)=\widehat{u}_{\mathrm{p}}(z) \exp (\widehat{\sigma} \widehat{t}) \\
& \widehat{T}_{\mathrm{P}}(z, t)=\widehat{\theta}_{\mathrm{p}}(z) \exp (\widehat{\sigma} \widehat{t})
\end{aligned}
$$

with: $\widehat{z}=z / \delta, \widehat{U}_{\mathrm{P}}=U_{\mathrm{P}} / u_{* \mathrm{eq}}, \widehat{T}_{\mathrm{P}}=T_{\mathrm{P}} / \theta_{* \mathrm{eq}}, \widehat{t}=t u_{* \mathrm{eq}} / \delta$ and $\widehat{\sigma}=\sigma \delta / u_{* \mathrm{eq}}$.

The governing equations for these perturbations are derived in Appendix B. This finally gives:

$$
\begin{gathered}
\frac{\partial \widehat{U}_{\mathrm{P}}}{\partial \widehat{t}}=\kappa \frac{\partial}{\partial \widehat{z}}\left(\widehat{z} \frac{2+4 b \widehat{z}}{1+b \widehat{z}} \frac{\partial \widehat{U}_{\mathrm{P}}}{\partial \widehat{z}}\right)-2 \kappa \frac{\partial}{\partial \widehat{z}}\left(\widehat{z} \frac{b \widehat{z}}{1+b \widehat{z}} \frac{\partial \widehat{T}_{\mathrm{P}}}{\partial \widehat{z}}\right) \\
\frac{\partial \widehat{T}_{\mathrm{P}}}{\partial \widehat{t}}=\kappa \frac{\partial}{\partial \widehat{z}}\left(\widehat{z} \frac{1+4 b \widehat{z}}{1+b \widehat{z}} \frac{\partial \widehat{U}_{\mathrm{P}}}{\partial \widehat{z}}\right)+\kappa \frac{\partial}{\partial \widehat{z}}\left(\widehat{z} \frac{1-2 b \widehat{z}}{1+b \widehat{z}} \frac{\partial \widehat{T}_{\mathrm{P}}}{\partial \widehat{z}}\right)
\end{gathered}
$$

with: $b=\alpha \delta / L_{\mathrm{eq}}$.

Inserting the exponential type of perturbations and dividing the results by $\exp (\widehat{\sigma} \widehat{t})$ leads to two coupled ordinary differential equations for $\widehat{u}_{\mathrm{p}}(z)$ and $\widehat{\theta}_{\mathrm{p}}(z)$ :

$$
\begin{aligned}
& \widehat{\sigma} \widehat{u}_{\mathrm{P}}=\kappa \frac{\partial}{\partial \widehat{z}}\left(\widehat{z} \frac{2+4 b \widehat{z}}{1+b \widehat{z}} \frac{\partial \widehat{u}_{\mathrm{P}}}{\partial \widehat{z}}\right)-2 \kappa \frac{\partial}{\partial \widehat{z}}\left(\widehat{z} \frac{b \widehat{z}}{1+b \widehat{z}} \frac{\partial \widehat{\theta}_{\mathrm{P}}}{\partial \widehat{z}}\right) \\
& \widehat{\sigma} \widehat{\theta}_{\mathrm{P}}=\kappa \frac{\partial}{\partial \widehat{z}}\left(\widehat{z} \frac{1+4 b \widehat{z}}{1+b \widehat{z}} \frac{\partial \widehat{u}_{\mathrm{P}}}{\partial \widehat{z}}\right)+\kappa \frac{\partial}{\partial \widehat{z}}\left(\widehat{z} \frac{1-2 b \widehat{z}}{1+b \widehat{z}} \frac{\partial \widehat{\theta}_{\mathrm{P}}}{\partial \widehat{z}}\right)
\end{aligned}
$$

The perturbations can only exist if they fulfill both the perturbation equations and the boundary conditions. This puts a restriction on $\widehat{\sigma}$ : for each set of external parameters (i.e. for each set of $U_{\mathrm{TOP}}, H_{0}, \delta$ and $z_{0}$ ) there is a corresponding $\widehat{\sigma}$ in order that the solution may exist. Let us assume that a perturbation of the exponential type (no matter how small) is actually present, then obviously the sign of (the real part) of $\widehat{\sigma}$ will determine what will happen to this disturbance. If $\widehat{\sigma}<0$, a small disturbance disappears in time, and the equilibrium state is said to be stable. Likewise, $\widehat{\sigma}>0$ indicates instability of the equilibrium solution.

In principle, the general method above enables us to calculate $\widehat{\sigma}$ so that local stability information is gained for all equilibrium situations, fixed by the imposed external 
parameters. In the present case, this is not a trivial task, due to the structure of the equations, showing explicit $\widehat{z}$-dependence in the coefficients. Therefore, we choose to determine the so-called marginal curve (i.e. the $\widehat{\sigma}=0$ curve) between the parameters (e.g. [18]). It is then assumed that there is an exchange of stability and that the marginal curve separates stability from instability. The validity of the assumption is verified by comparison with the numerical results.

\subsubsection{Solution of the perturbation equations}

The 1.h.s. of the Eqs. 18 and 19 is set to zero to find the governing equation for the marginal state. In order to find a solution, the equations are transformed to a standard problem by linearly combining the equations, $18-19$ and $18-\frac{1}{2} 19$, using the substitution variables: $\widehat{u}_{\mathrm{p}}-\widehat{\theta}_{\mathrm{P}}=\widehat{k}_{\mathrm{P}}$ and $\widehat{u}_{\mathrm{p}}-\frac{1}{2} \widehat{\theta}_{\mathrm{P}}=\widehat{n}_{\mathrm{P}}$. Finally, the solution reads:

$$
\widehat{u}_{\mathrm{p}}(\widehat{z})=C_{1} \ln \widehat{z}+C_{2}\left(\frac{\widehat{z}}{2}-\frac{\ln \widehat{z}}{2 b}\right)+C_{3} \quad \widehat{\theta}_{\mathrm{p}}(\widehat{z})=C_{1} \ln \widehat{z}+C_{2} \widehat{z}+C_{4}
$$

The four constants $C_{1}$ to $C_{4}$ have to be fixed by four constraints from our boundary conditions (Section 3.1). Before we apply the boundary conditions (BCs) it is noted that the perturbation fluxes are found from back substitution of the solutions:

$$
\tau_{\mathrm{p}}=2 C_{1}-C_{2} / b \quad H_{\mathrm{p}}=2 C_{1}-C_{2} /(2 b)
$$

The four boundary conditions of the perturbations in the marginal state:

$$
\begin{array}{ll}
\widehat{u}_{\mathrm{p}}\left(\widehat{z}=\widehat{z}_{0}\right)=0 & \widehat{u}_{\mathrm{p}}(\widehat{z}=1)=0 \\
H_{\mathrm{p}}\left(\widehat{z}=\widehat{z}_{0}\right)=0 & \widehat{\theta}_{\mathrm{p}}(\widehat{z}=1)=0
\end{array}
$$

Thus we have four equations with four unknowns. This has only a nontrivial solution when the determinant of the system is equal to zero (eigenvalue problem). Or, alternatively, by subsequent substitution of the BCs this leads to the condition: $C_{2}\left(-\frac{\ln \widehat{z}_{0}}{4 b}+\frac{2 b\left(\widehat{z_{0}}-1\right)}{4 b}\right)=0$, which has only a nontrivial solution when the parameters are related according to (backsubstitution of $b=\alpha \delta / L_{\mathrm{eq}}$ and $\widehat{z}_{0}=z_{0} / \delta$ ):

$$
\left.\frac{\delta}{L_{\text {eq }}}\right|_{\text {MARGINAL }}=\frac{\ln \left(\frac{\delta}{z_{0}}\right)}{2 \alpha\left(1-\frac{z_{0}}{\delta}\right)}
$$

This relation characterizes the so-called marginal state of the system. It is an important result, because this state separates unstable from stable solutions and, as such, the criterion serves as a predictor of the system stability for numerical simulations. Comparison of Eq. 15 with Eq. 23 learns that the transition from stable to unstable 
occurs exactly at the turning point of the equilibrium solution (Fig. 6). In the next section this theoretical result is compared with numerical simulations.

\section{Comparison Analytical and Numerical Results}

To verify the theoretical prediction that the system changes stability at the turning point, we run the numerical simulation from three different initial conditions, for the same external forcings, with $H_{0}=-10\left[\mathrm{~W} \mathrm{~m}^{-2}\right]$ and $U_{\mathrm{TOP}}=4$ [ms $\left.{ }^{-1}\right]$ (Fig. 7). Cases A, B and $\mathrm{C}$ are chosen such that they depart from different sides of the equilibrium line. The initial values read: Case A $\widehat{u}_{*}=1.0$, Case B $\widehat{u}_{*}=0.5$ and Case C $\widehat{u}_{*}=0.4$ (the initial $u_{*}$ values are found by multiplication with $u_{*_{N}}=0.29$ ) and $-\widehat{H}=0.1$.

Figure 8 shows that both case $\mathrm{A}$ and $\mathrm{B}$ are attracted to the same equilibrium solution, which corresponds to the upper (stable) branch of Fig. 7. However, case C, starting just below the lower branch, shows a definite collapse. This interesting fact implies that, in principle, the predictability of our simple system is limited depending on initial conditions (in fact similar to McNider et al. [25]). In practice however, the atmospheric SBL usually grows from a neutral initial state as a consequence of the day-night transition, so that a SBL evolution according to Figs. 2 and 3 is most likely to occur. A multitude of numerical runs were made, all showing behaviour similar to cases A, B and C. From this it is concluded that the upper branch of the equilibrium diagram is stable and that the lower branch is unstable, with the marginal state at the turning point, as predicted by the theoretical analysis. The results are summarized in Fig. 9, which is a bifurcation diagram rather than an equilibrium diagram in a sense that also the stability of the equilibrium line is indicated as: stable (solid) and unstable (dashed), following the conventions in nonlinear system analysis (e.g. [35]).

Finally, we compare the predictions by the analytical expression for the marginal state in Eqs. 23 and 15, with the numerical simulation of the collapse phenomenon in Fig. 2. In Fig. 10 the value of $\delta / L_{\text {eq }}$ in the marginal state/turning point is plotted against $z_{0} / \delta$ according to Eqs. 23 and 15. A general situation is analyzed as follows. For given $\left(U_{\mathrm{TOP}}\right.$, $H_{0}, \delta$ and $z_{0}$ ) two values of $u_{*}$ eq are found according to (11). This leads to two values of $\delta /$ $L_{\text {eq }}$ : one with a $\delta / L_{\text {eq }}$ larger than the critical value (the unstable branch), and one with a

Fig. 7 Initial conditions (crosses) of the selected cases A, B and C (see: text)

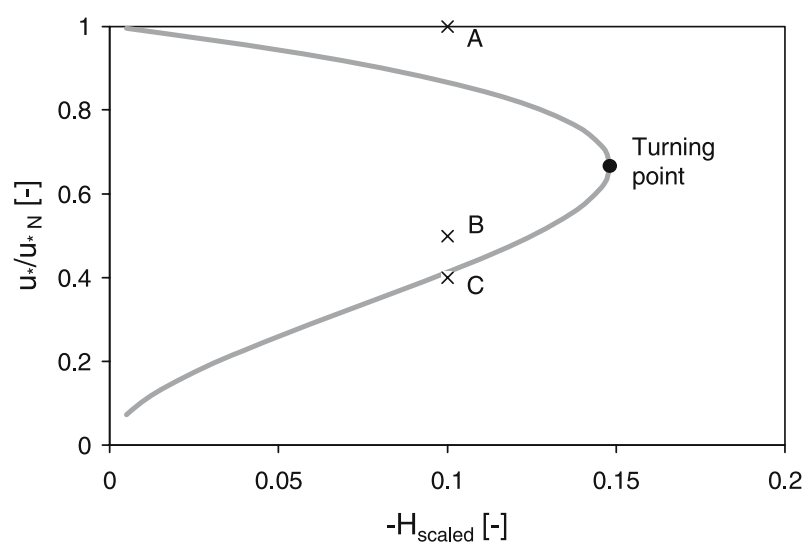


Fig. 8 Transient simulation of cases $\mathrm{A}, \mathrm{B}$ and $\mathrm{C}$ corresponding to the initial conditions indicated in Fig. 7

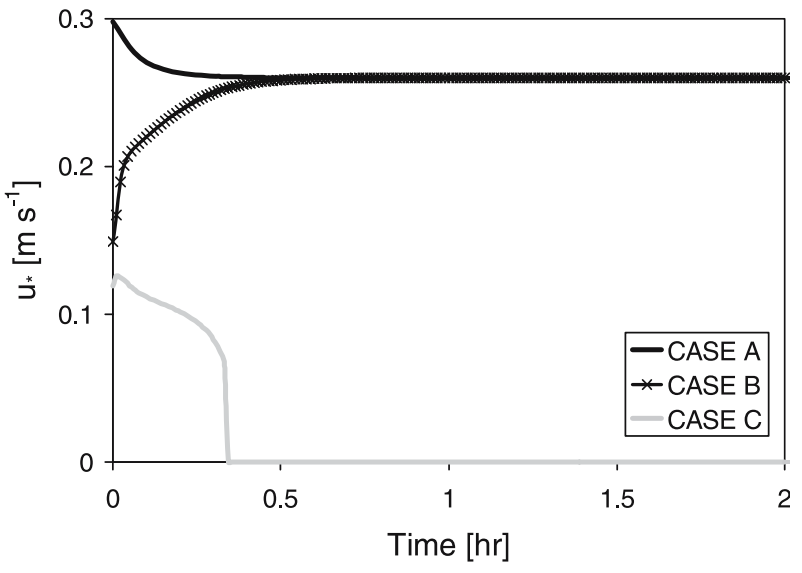

lower value (the stable branch). Of course, the stable solution is the one that will be observed, i.e. when the simulation is started from neutral initial conditions. As the cooling is increased the two solutions will approach each other until both values of $\delta / L$ are equal to the critical value $\delta / L_{T}$ in the marginal/turning point. A further increase in the cooling (i.e. larger $\delta / L$ ) then results in a collapse of the system, because no equilibrium exists at this cooling rate (arrow at right end in Fig. 9).

Next, the theoretical predictions by Fig. 10 are verified from the example introduced in Section 2. In the example $\delta=23.6[\mathrm{~m}]$ and $z_{0}=0.1[\mathrm{~m}]$ leading to $\delta / L_{T}=0.55[-]$ as the predicted critical value for continuous turbulence. Comparison with actual numerical simulations in Fig. 2 learns that the predicted $\delta / L_{T}=0.55$ indeed closely corresponds to the $\delta / L$ value in the limiting case, where turbulence just survives $(0.52)$. The predictions were verified for a large number of cases with variable values of $\left(U_{\mathrm{TOP}}, H_{0}, \delta\right.$ and $\left.z_{0}\right)$ and variable time steps and showed all to be in agreement.

In summary: the validity of the analytical framework based on linear stability analysis, is verified by transient numerical simulations with the full time-dependent nonlinear model. As such quantitative predictions on the collapse of turbulence can be made directly from evaluation of the forcing parameters $\left(U_{\text {TOP }}\right.$ and $\left.H_{0}\right)$.

Fig. 9 Bifurcation diagram. The solid line represents the upper (stable) branch of the equilibrium solution (Eq. 11), and the dashed line the lower (unstable) branch. The black dot represents the marginal/turning point (saddle node). The time dependent behaviour is indicated by the arrows

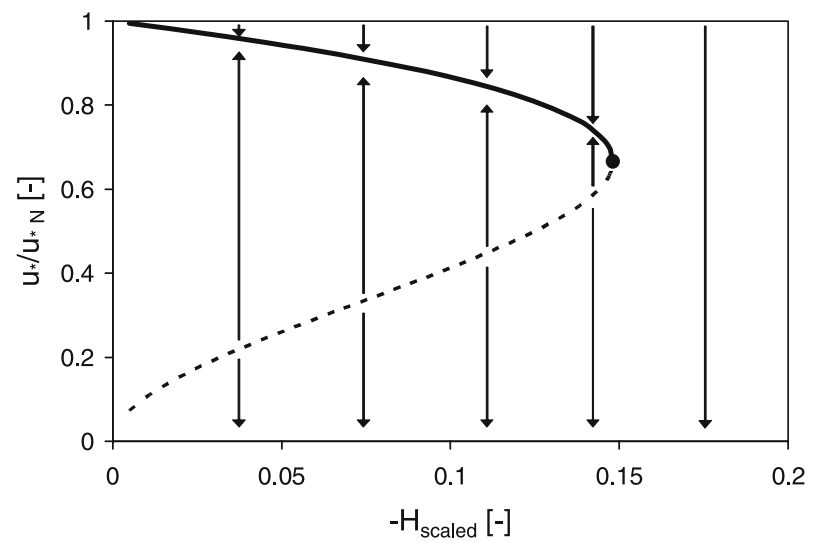


Fig. 10 Stability diagram. Relation between $\delta / L$ and $z_{0} / \delta$ at marginal state/turning point according to Eqs. (23)/(15). The areas with continuous turbulence and with collapsed turbulence are indicated. Also the predicted critical value of $\delta / L$ for the example in Section 2 is given. Note the limit to the $z_{0}$ - concept for large value of $z_{0} / \delta$ (dashed line)

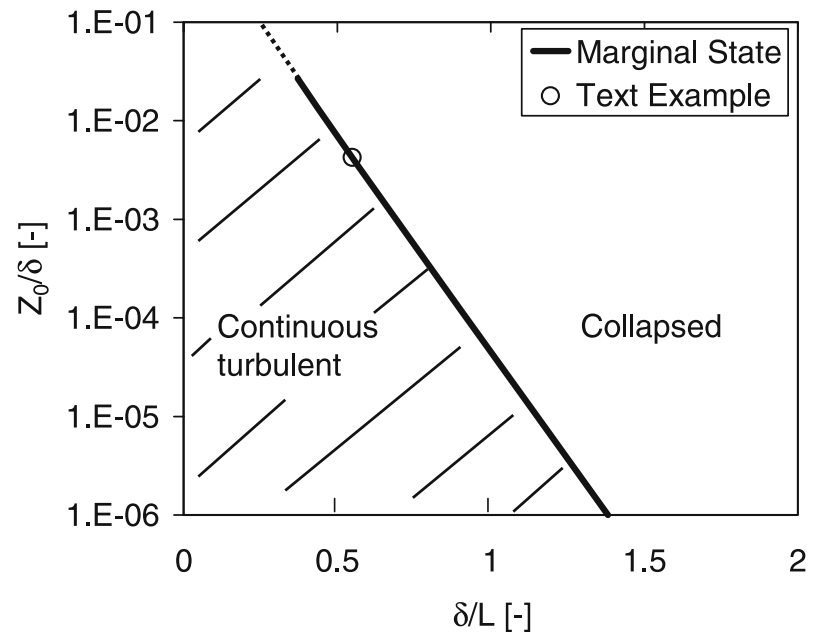

\section{Discussion}

5.1 Extension to the atmospheric case: the impact of boundary conditions

For current configuration, the theoretical framework enables prediction of the collapse phenomenon. As a next step, the theoretical predictions could be compared directly with more advanced turbulence models such as direct numerical simulation models (DNS) or with laboratory data, using the same boundary and forcing conditions. Such a comparison may indicate merits and limitations of the theory and the methods used.

However, in line of the meteorological background of the collapse phenomenon indicated in the introduction, it is tempting to have some thoughts on the possibility of extending the theory to configurations encountered in the atmosphere. Therefore, in Fig. 11 our bifurcation diagram is shown (as Fig. 10) together with observations from the atmospheric CASES99 field experiment. The experiment took place during the whole month of October 1999, near Wichita, Kansas, USA. The experimental area, covered with prairie-grass, is relatively flat. A vast array of instruments was deployed (Poulos et al. [33].

Fig. 11 As in Fig. 10, with observational data from the CASES99 field experiment

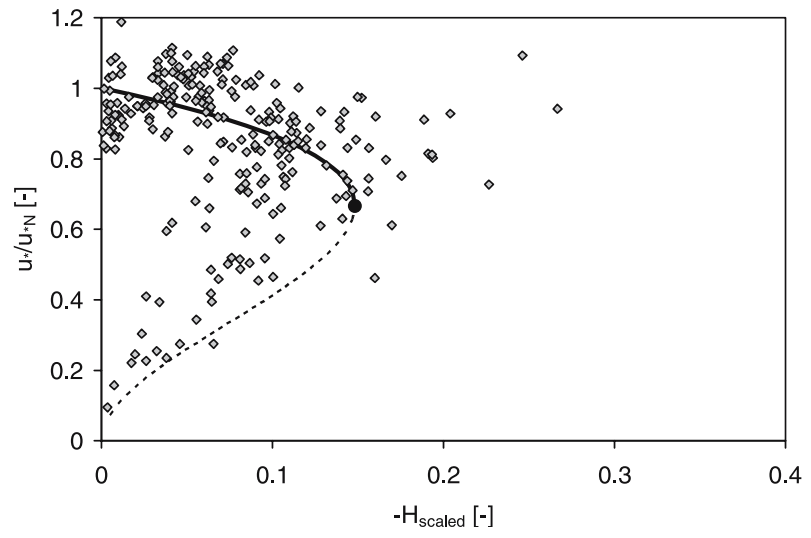


Wageningen University carried out observations at one point in a nested network of flux stations around the central 55-m flux tower of NCAR. An eddy covariance system was setup at heights of 2.65 and $10.1 \mathrm{~m}$ and operated at $20 \mathrm{~Hz}$. It consisted of a CSAT3 sonic anemometer and a $\mathrm{KH} 2 \mathrm{O}$ Krypton hygrometer. Raw data were processed as described in Hartogensis et al. [13].

From the wind speed and the turbulent flux observation (5 min avg.), scaled variables are calculated according to Eq. 11. Night data from the whole month are used. To ensure reasonable stationarity, the data were filtered in a sense that only observations are shown where difference between the $2.65 \mathrm{~m}$ and the $10.1 \mathrm{~m}$ level fluxes was less than $15 \%$. However, we note that the filtering did not change the results dramatically. The height of our system $\delta$ extends to the highest observational level, $\delta=10.1[\mathrm{~m}]$, and based on earlier findings $z_{0}$ is set equal to 0.03 [m] [41].

Although the scattering of the data in Fig. 11 is considerable, the data seem to follow the equilibrium line reasonable. To some extent this could be expected, because it is a direct theoretical consequence of the validity of a log-linear behaviour of Monin-Obukhov similarity, which is well established at least for weak to moderate stratification (Section 2). An interesting result is the fact that data points close to the unstable branch are found. A closer look at individual nights reveals that these 'unstable' cases move in time along the dashed line, in stead of vertical as in Fig. 10. This is related to the fact, that in our theoretical model configuration, the turbulent heat flux at the surface is fixed, whereas in the atmosphere, a collapse of turbulence (as indicated by a collapse of friction velocity) directly implies a collapse of the turbulent heat flux. Finally, we mention the observed points along the stable branch (solid line) that tend to appear in clusters for individual nights (not shown), which reflects the fact that those situations are more or less 'stable' in time.

It is concluded that the present theoretical framework can only be applied to atmospheric studies, when the surface boundary condition is changed accordingly. In fact, such an extension could readily be achieved by including a simple surface energy balance (SEB) equation, so that the surface heat flux is calculated as an internal variable rather than a forcing parameter. Note that, in case of a SEB, (negative) feedbacks are expected from the soil heat flux and the long wave radiation processes, as mentioned by Derbyshire $[7,8]$ and Van de Wiel et al. [40].

For application purposes also the upper boundary condition needs attention: unlike in our model configuration, the wind speed and the temperature are generally not fixed at a certain height in the atmosphere. With the perturbations vanishing at this height $\delta$, the formal stability criterion (Eq. 23) directly depends on this particular choice for $\delta$ (of course in a laboratory or DNS this $\delta$ could be prefixed). However, for the atmosphere, predicted instability then depends on this particular choice of $\delta$. It is clear that such a criterion would be rather subjective, unless $\delta$ is taken 'far enough' from the near surface perturbations, so that the criterion is not very sensitive to the exact value of $\delta$. In fact, this is often the case, as long as $\delta \geq \mathrm{O}(10)[\mathrm{m}]$ and $z_{0}<<1[\mathrm{~m}]$, as shown with the following numerical example: with $z_{0}=0.03[\mathrm{~m}]$, and values for $\delta$ of 10,20 and $40[\mathrm{~m}]$ the resulting $\delta / L_{\text {critical }}$ will be $0.58,0.65$ and 0.72 respectively. Thus, taking into account the formal limitations, it may be useful to compare the predictions of turbulence collapse to observations that could be related to this phenomenon. In the literature transitions in SBL flow behaviour are reported to occur at $z / L$ of O( 0.5-0.7) (Smedman [36], Högström [14] and Mahrt et al. [23]). Inspired by the work of Smedman, Mahrt et al. [23] show that the normalized standard deviation of horizontal motions suddenly increases for values of $z / L$ larger than 0.6. This seems to indicate a strong change in the character of the flow, which could be related to the collapse phenomenon in the present study at similar $\delta / L$ values. 
Apart from comparison with near-surface observations such as indicated above, it is challenging to predict the collapse of the atmospheric boundary as a whole, that is over the total depth of the turbulent boundary layer $h$. In that context, interesting observations at Cabauw are reported by Holtslag and Nieuwstadt [15] who state: "For stable conditions, however, no fully turbulent $A B L$ 's are observed in Cabauw data beyond $h / L \cong 6$ ”. Of course, in a full atmospheric SBL the flow forcing is different (pressure driven) and Coriolis effects are present, which is significantly different from our flow configuration (see below). Furthermore, many complicating factors, especially longwave radiative transfer and gravity wave activity, may modulate local Ri and thus affect local turbulent activity (e.g. [17]).

In summary: our couette flow $\delta / L$ criterion cannot be compared directly to the atmospheric critical $h / L$-value. Nevertheless, the fact that the SBL seems to collapse at particular threshold-value of $h / L$ encourages future application of the present framework to atmospheric flow conditions.

\subsection{Collapse of turbulence in direct numerical simulation}

In the context of the present work and of this special issue in particular, we would like to mention the recent results of Nieuwstadt ([32]; from now on N05), in studying the collapse of turbulence in the SBL by means of direct numerical simulation (DNS). In line with our results, Nieuwstadt's results show a definite collapse of turbulence when a certain critical value of $\delta / L$ is exceeded. In his case this particular $\delta / L_{\text {critical }}=1.25$. Again, this critical value itself may not be compared quantitatively to predictions by Eqs. 23, because of the different model configuration in N05. In N05 the mechanical forcing is supplied by a pressure gradient with a free-slip condition at the model top, whereas in our case the wind speed at the top is fixed. Also, in the DNS an aerodynamically smooth flow is simulated instead of rough flow (present study). The practical assumption that $z_{0} \approx 0.135 \nu / u_{*}$ (e.g. [31]), with very small $z_{0}$, may lead to the misleading conclusion that $z_{0} / \delta$ is small and thus $\delta / L_{\text {critical }} \approx O$ (1) (Fig. 6), in accordance with the DNS simulations. In fact $\delta$ is usually small too in DNS, suggesting $\delta / L_{\text {critical }}<1$, different from N05 results due to a different model configuration (see below). It is important to note that, for smooth flow $z_{0} / \delta \propto 1 / \operatorname{Re}_{*}$, so that our scaling diagram contains the shear Reynolds number in stead of roughness on the vertical axis!

In any case, the philosophy of the present work and the method of Section 3 could be adapted to the DNS model configuration of Nieuwstadt [32] to enable a quantitative comparison in the future. The success of the method depends quite heavily on the closure assumption in the governing Eqs. 1-4. In this perspective a very interesting and innovative result was presented by N05. In his Fig. 15b the eddy diffusivity as inferred from the stationary DNS results, is compared to the eddy diffusivity from the local closure assumption (our Eq. 3). The correspondence is surprisingly good, and suggests some generality of Eq. 3 over a large range of Re (Re similarity): the original relationship was derived from atmospheric flows with much larger Re than in the DNS study of N05.

N05 uses the closure indicated above to construct an analytical equilibrium model, as in Section 3. A 'surprising' result is found, namely that $\mathrm{Ri}<R_{\mathrm{c}}$ in large part of the channel, for all values of $\delta / L$ (Note: N05 defines a parameter $B$ proportional to $L / \delta$ ). Then Nieuwstadt concludes that this implies a continuous turbulent solution for all $\delta / L$, in contradiction with his numerical findings: "The fact that for the range $0<B<\infty$ there is always one solution for a continuously turbulent stationary flow, seems to be also in contradiction with the results of Section 3 where we found an indication that for $\delta / L \geq 1.25$ no stationary turbulence exists. Our closure model with $f(R i)$ cannot explain this critical behaviour near $\delta / L=1.25 "$. 
In line of the results of the present paper, we unfortunately cannot agree with the statement above. Clearly, it is not the equilibrium itself but also the stability of this equilibrium boundary layer as a whole that determines the eventual state of the system. Again, also in the limiting case of Fig. 2, with $\delta / L_{\text {eq }} \approx 0.52$ the Richardson number is lower than its critical value prior to the collapse, because in any case:

$$
\operatorname{Ri}_{\mathrm{eq}}(z)=R_{\mathrm{c}} \frac{z / L_{\mathrm{eq}}}{z / L_{\mathrm{eq}}+R_{\mathrm{c}}}<R_{\mathrm{c}}
$$

Thus the present analysis provides a logical explanation for the paradoxical result obtained in N05. The authors agree with N05 that after this collapse of turbulence a laminar flow may develop which could, in turn, be subjected to stability analysis. In our case the stability of the laminar flow (a plane Couette flow), is in that case indeed determined by the classical Ri-criterion for stability of laminar flow $\left(\mathrm{Ri}<\frac{1}{4}\right)$, as noted by Miles [26]. When this laminar solution is also hydrodynamically unstable, a kind of 'switching' between turbulent and laminar states may result leading to an intermittent turbulent flow.

\section{Conclusions}

We studied the collapse of turbulence in a plane channel flow model as a simple flow analogy of stably stratified atmospheric flow. Turbulence is parameterized by first order closure and the surface heat flux is prescribed as are temperature and wind speed at model top. The model is studied both by using numerical simulations and by using linear stability analysis. The results lead to the following conclusions:

- - For a given roughness to channel depth ratio $z_{0} / \delta$, the numerical simulations (with neutral initial conditions) show a definite collapse of turbulence when a certain value of the critical parameter $\delta / L$ is exceeded.

- $\quad-$ The method introduced by Derbyshire [7, 8] to predict the stability of a turbulent linearly stratified shear flow is generalized to the more realistic case of log-linear stratification. The method is nonclassical in a sense that the stability of a parameterized set of equations of a turbulent flow is analyzed instead of a particular laminar flow solution.

- - Our theory predicts a collapse of turbulence at $\delta / L$ between 0.5 and 1 , depending on the particular ratio $z_{0} / \delta$. The predictions are in good agreement with the results of the numerical simulations.

- - It is shown that a general overview of the model behaviour is readily obtained by a system dynamics approach to the problem using a bifurcation diagram. In the diagram both the equilibrium behaviour, the stability of the equilibria and sensitivity of the results to initial conditions are depicted.

- -The present study shows a qualitative agreement with results from direct numerical simulation by Nieuwstadt [32] in a sense that a collapse of turbulence occurs at a particular value of $\delta / L$ (i.e. for given $z_{0} / \delta$ (rough) or $\mathrm{Re}_{*}$ (smooth flow)). A direct quantitative comparison is not possible, because of the different model configurations (and boundary conditions) used in both studies.

- - Interestingly, the present study seems to solve the paradox in Nieuwstadt [32]: We show that in our case $\mathrm{Ri}_{\mathrm{eq}}(z)<R_{\mathrm{c}}$ for all $z$, at the collapse of turbulence. Thus, the fact that $\operatorname{Ri}_{\text {eq }}(z)<R_{\mathrm{c}}$ in our system does not imply that the boundary layer is able to 
maintain its continuous turbulent character. On the contrary, the collapse of turbulence that occurs for $\operatorname{Ri}_{\text {eq }}(z)<R_{\mathrm{c}}$ can be explained naturally as a linear instability of the system.

- $\quad$ The present framework enables a direct comparison with DNS/laboratory studies of the collapse phenomenon for the flow configuration presented.

- -Although promising results are obtained in comparison with atmospheric data, a good quantitative comparison can only be made, when the present theoretical framework is extended to the particular forcing/boundary conditions that are valid in atmospheric stratified flows.

Acknowledgements We are indebted to Prof. Frans Nieuwstadt for his inspiring work on stable boundary layers and for the fruitful discussions in previous years. Our colleague Dr. Henk de Bruin is acknowledged for his earlier personal communication on the 'duality' of stable boundary layers, which certainly influenced the present work. Finally, the contributions of Dr. Mark van de Wiel on a mathematical issue and discussions with Dr. Van Herwaarden and Dr. Gielen (Wageningen Univ.) in an early stage of the research are highly appreciated.

\section{Appendix A}

Derivation of Equilibrium Profiles

In a stationary situation 7 and 8 become

$$
\begin{gathered}
u_{*}^{2}=(\kappa z)^{2}(\partial U / \partial z)^{2}\left(1-\mathrm{Ri} / R_{\mathrm{c}}\right)^{2} \\
\theta_{*} u_{*}=(\kappa z)^{2}(\partial T / \partial z)(\partial U / \partial z)\left(1-\mathrm{Ri} / R_{\mathrm{c}}\right)^{2}
\end{gathered}
$$

Combining and introducing $\alpha=1 / R_{\mathrm{c}}$ gives:

$$
u_{*}^{2} / \theta_{*}=(\kappa z) \frac{(\partial U / \partial z)^{2}}{\partial T / \partial z}(1-\alpha \mathrm{Ri})
$$

multiplication by $T_{\text {ref }} / g$ and rearranging gives:

$$
\frac{z}{L}=\frac{\mathrm{Ri}}{1-\alpha \mathrm{Ri}} \text { or }(1-\alpha \mathrm{Ri})=\frac{1}{1+\alpha z / L} \text { with }: L \equiv \frac{u_{*}^{2}}{\theta_{*}} \frac{T_{\text {ref }}}{\kappa g}
$$

The latter result, which expresses $(1-\alpha \mathrm{Ri})$ in terms of $z / L$ is substituted in the definitions of $u_{*}$ and $\theta_{*}$ above which gives:

$$
\frac{\partial U}{\partial z}=\frac{u_{*}}{\kappa z}\left(1+\alpha \frac{z}{L}\right) \text { and } \frac{\partial T}{\partial z}=\frac{\theta_{*}}{\kappa z}\left(1+\alpha \frac{z}{L}\right)
$$

Finally, integration with respect to $z$ leads to expressions 9 and 10 . 


\section{Appendix B}

Derivation of the Perturbation Equations

In abstract form our governing equations read:

$$
\begin{aligned}
& \frac{\partial U}{\partial t}=f_{1}(\partial U / \partial z, \partial T / \partial z) \\
& \frac{\partial T}{\partial t}=f_{2}(\partial U / \partial z, \partial T / \partial z)
\end{aligned}
$$

The system's behaviour near the equilibrium is studied by linearization of the equations:

$$
\begin{aligned}
\frac{\partial U}{\partial t} & =f_{1} \approx f_{\text {leq }}+\left.\frac{\partial f_{1}}{\partial(\partial U / \partial z)}\right|_{\mathrm{eq}}\left((\partial U / \partial z)-(\partial U / \partial z)_{\mathrm{eq}}\right) \\
& +\left.\frac{\partial f_{1}}{\partial(\partial T / \partial z)}\right|_{\mathrm{eq}}\left((\partial T / \partial z)-(\partial T / \partial z)_{\mathrm{eq}}\right) \\
\frac{\partial T}{\partial t} & =f_{2} \approx f_{2 \mathrm{eq}}+\left.\frac{\partial f_{2}}{\partial(\partial U / \partial z)}\right|_{\mathrm{eq}}\left((\partial U / \partial z)-(\partial U / \partial z)_{\mathrm{eq}}\right) \\
& +\left.\frac{\partial f_{2}}{\partial(\partial T / \partial z)}\right|_{\mathrm{eq}}\left((\partial T / \partial z)-(\partial T / \partial z)_{\mathrm{eq}}\right)
\end{aligned}
$$

We realize that $f_{1 \mathrm{eq}}=0=f_{2 \mathrm{eq}}$ and that we may subtract $\partial U_{\mathrm{eq}} / \partial t=0=\partial T_{\mathrm{eq}} / \partial t$ on the 1.h.s. As such the governing equations for small perturbation are found. Application to our system, Eqs. 7 and 8, gives:

$$
\begin{gathered}
\frac{\partial U_{\mathrm{P}}}{\partial t}=\frac{\partial}{\partial z}(\kappa z)^{2} x_{\mathrm{eq}}\left[2 f-2 \mathrm{Ri} f^{\prime}\right]_{\mathrm{eq}} x_{\mathrm{p}}+\frac{\partial}{\partial z}(\kappa z)^{2} x_{\mathrm{eq}} \frac{x_{\mathrm{eq}}}{y_{\mathrm{eq}}}\left[R i f^{\prime}\right]_{e q} y_{p} \\
\frac{\partial T_{\mathrm{P}}}{\partial t}=\frac{\partial}{\partial z}(\kappa z)^{2} x_{\mathrm{eq}} \frac{y_{\mathrm{eq}}}{x_{\mathrm{eq}}}\left[f-2 \mathrm{Ri} f^{\prime}\right]_{\mathrm{eq}} x_{\mathrm{p}}+\frac{\partial}{\partial z}(\kappa z)^{2} x_{\mathrm{eq}}\left[f+\mathrm{Ri} f^{\prime}\right]_{\mathrm{eq}} y_{\mathrm{p}}
\end{gathered}
$$

with: $x=\partial U / \partial z$ and $y=\partial T / \partial z$ and the subscript ' $\mathrm{P}$ ' indicates the perturbed variable, e.g. $x_{\mathrm{p}}=x-x_{\text {eq }}$. Also the stability function $f=f(\mathrm{Ri})$ and $f^{\prime}=d f(\mathrm{Ri}) / d \mathrm{Ri}$.

It is realized that equilibrium gradients in the equations above are directly related to the equilibrium fluxes via differentiation of Eqs. 9 and 10. This, finally, leads to the scaled version of our perturbation equations:

$$
\begin{aligned}
& \frac{\partial \widehat{U}_{\mathrm{P}}}{\partial \widehat{t}}=\kappa \frac{\partial}{\partial \widehat{z}} \widehat{z} \frac{2+4 b \widehat{z}}{1+b \widehat{z}} \frac{\partial \widehat{U}_{\mathrm{P}}}{\partial \widehat{z}}-2 \kappa \frac{\partial}{\partial \widehat{z}} \widehat{z} \frac{b \widehat{z}}{1+b \widehat{z}} \frac{\partial \widehat{T}_{\mathrm{P}}}{\partial \widehat{z}} \\
& \frac{\partial \widehat{T}_{\mathrm{P}}}{\partial \widehat{t}}=\kappa \frac{\partial}{\partial \widehat{z}} \widehat{z} \frac{1+4 b \widehat{z}}{1+b \widehat{z}} \frac{\partial \widehat{U}_{\mathrm{P}}}{\partial \widehat{z}}+\kappa \frac{\partial}{\partial \widehat{z}} \widehat{z} \frac{1-2 b \widehat{z}}{1+b \widehat{z}} \frac{\partial \widehat{T}_{\mathrm{P}}}{\partial \widehat{z}}
\end{aligned}
$$

With $b=\alpha \delta / L_{\mathrm{eq}}, \widehat{z}=z / \delta, \widehat{U}_{\mathrm{P}}=U_{\mathrm{P}} / u_{* \mathrm{eq}}, \widehat{T}_{\mathrm{P}}=T_{\mathrm{P}} / \theta_{* \mathrm{eq}}$ and $\widehat{t}=t u_{* \mathrm{eq}} / \delta$. This result will be used further on. 


\section{References}

1. Basu, S., Porté-Agel, F., Foufoula-Georgiou, E., Vinuesa, J.-F., Pahlow, M.: Revisiting the local scaling hypothesis in stably stratified atmospheric boundary layer turbulence: An integration of field and laboratory measurements with large-eddy simulations. Bound. Layer Meteor. 119, 473-500 (2006)

2. Businger, J.A., Wyngaard, J.C., Izumi, Y., Bradley, E.F.: Flux-profile relationships in the atmospheric boundary layer. J. Atmos. Sci. 30, 788-794 (1971)

3. Chandrasekhar, S.: Hydrodynamic and Hydromagnetic Stability. Clarendon, Oxford (1961)

4. De Bruin, H.A.R.: Analytic solutions of the equations governing the temperature fluctuation method. Bound. Layer Meteor. 68, 427-432 (1994)

5. Derbyshire, S.H.: Nieuwstadt's stable boundary layer revisited. Q. J. R. Meteorol. Soc. 116, 127-158 (1990)

6. Derbyshire, S.H.: A "balanced" approach to stable boundary layers. J. Atmos. Sci. 51, 3486-3504 (1994)

7. Derbyshire, S.H.: Stable boundary layer modelling: Established approaches and beyond. Bound. Layer Meteor. 90, 423-446 (1999a)

8. Derbyshire, S.H.: Boundary-layer decoupling over cold surfaces as a physical boundary instability. Bound. Layer Meteor. 90, 297-325 (1999b)

9. Drazin, P.G., Reid, W.H.: Hydrodynamic Stability. Cambridge University Press (2004)

10. Duynkerke, P.G., De Roode, S.R.: Surface energy balance and turbulence characteristics observed at the SHEBA Ice Camp during FIRE III. J. Geophys. Res. 106, 15313-15322 (2001)

11. Garratt, J.R.: The Atmospheric Boundary Layer. Cambridge University Press (1992)

12. Hanazaki, H., Hunt, J.C.R.: Structure of unsteady stably stratified turbulence with mean shear. J. Fluid Mech. 507, 1-42 (2004)

13. Hartogensis, O.K., De Bruin, H.A.R., Van de Wiel, B.J.H.: Displaced-beam small aperture scintillometer test. Part II: CASES-99 stable boundary layer experiment. Bound. Layer Meteor. 105, 149-176 (2002)

14. Högström, U.: Review of some basic characteristics of the atmospheric surface layer. Bound. Layer Meteor. 78, 215-246 (1996)

15. Holtslag, A.A.M., Nieuwstadt, F.T.M.: Scaling the atmospheric boundary layer. Bound. Layer Meteor. 36, 201-209 (1986)

16. Howard, L.N.: Note on a paper of John W. Miles. J. Fluid Mech. 13, 158-160 (1961)

17. Hunt, J.C.R., Kaimal, J.C., Gaynor, J.E.: Some observations of turbulence structure in stable layers. Q. J. R. Meteorol. Soc. 111, 793-815 (1985)

18. Kundu, P.K.: Fluid Mechanics. Academic, San Diego (1990)

19. Lenschow, D.H., Li, X.S., Zhu, C.J., Stankov, B.B.: The stably stratified boundary layer over the great plains. Bound. Layer Meteor. 42, 95-121 (1988)

20. Louis, J.-F.: A parametric model of vertical eddy fluxes in the atmosphere. Bound. Layer Meteor. 17, 187-202 (1979)

21. Malhi, Y.S.: The significance of the dual solutions for heat fluxes measured by the temperature fluctuation method in stable conditions. Bound. Layer Meteor. 74, 389-396 (1995)

22. Mahrt, L.: Intermittency of atmospheric turbulence. J. Atmos. Sci. 46, 79-95 (1989)

23. Mahrt, L., Sun, J., Blumen, W., Delany, T., Oncley, S.: Nocturnal boundary layer regimes. Bound. Layer Meteor. 88, 255-278 (1998)

24. Mahrt, L.: Stratified atmospheric boundary layers. Bound. Layer Meteor. 90, 375-396 (1999)

25. McNider, R.T., England, D.E., Friedman, M.J., Shi, X.: Predictability of the stable atmospheric boundary layer. J. Atmos. Sci. 52, 1602-1614 (1995)

26. Miles, J.W.: On the stability of heterogeneous shear flows. J. Fluid Mech. 10, 496-508 (1961)

27. Monin, A.S., Obukhov, A.M.: Basic laws of turbulent mixing in the atmosphere near the ground. Tr. Akad. Nauk., SSSR Geophiz. Inst. 24(151), 1963-1987 (1954)

28. Nieuwstadt, F.T.M.: The turbulent structure of the stable, nocturnal boundary layer. J. Atmos. Sci. 41, 2202-2216 (1984)

29. Nieuwstadt, F.T.M.: A model for the stationary, stable boundary layer. In: Hunt, J.C.R. (ed.) Turbulence and Diffusion in Stable Environments, pp. 149-179. Clarendon (1985)

30. Nieuwstadt, F.T.M.: Turbulentie: inleiding in the theorie en toepassingen van turbulente stromingen (in Dutch). Epsilon Uitgaven, Utrecht (1992)

31. Nieuwstadt, F.T.M.: Turbulence and similarity theory in meteorology and engineering. Clear and cloudy boundary layers. In: Holtslag, A.A.M., Duynkerke, P.G., Jonker, P.J. (eds.) Royal Netherlands Ac. Of Arts and Sci., pp. 287-304. Amsterdam (1998) 
32. Nieuwstadt, F.T.M.: Direct numerical simulation of stable channel flow at large stability. Bound. Layer Meteor. 116, 277-299 (2005)

33. Poulos, G.S., Blumen, W., Fritts, D., Lundquist, J.L., Sun, J., Burns, S.P., Nappo, C., Banta, R., Newsom, R., Cuxart, J., Terradellas, E., Balsey, B., Jensen, M.: CASES-99: A comprehensive investigation of the stable nocturnal boundary layer. Bull. Am. Meteorol. Soc. 83, 555-581 (2002)

34. Schlichting, H., Gersten, K.: Boundary Layer Theory. Springer-Verlag Berlin, Heidelberg (2000)

35. Seydel, R.: From Equilibrium to Chaos: Practical Bifurcation and Stability Analysis. Elsevier (1988)

36. Smedman, A.-S.: Observations of a multi-level turbulence structure in a very stable atmospheric boundary layer. Bound. Layer Meteor. 44, 247-264 (1988)

37. Steeneveld, G.J., van de Wiel, B.J.H., Holtslag, A.A.M.: Modeling the evolution of the atmospheric boundary layer coupled to the land surface for three contrasting nights in CASES-99. J. Atmos. Sci. 63, 920-935 (2006)

38. Taylor, P.A.: A note on the log-linear velocity profile in stable conditions. Q. J. R. Meteorol. Soc. 97, 326-329 (1971)

39. Van de Wiel, B.J.H., Ronda, R.J., Moene, A.F., De Bruin, H.A.R., Holtslag, A.A.M.: Intermittent turbulence and oscillations in the stable boundary layer. Part I: A bulk model. J. Atmos. Sci. 59, 942-958 (2002a)

40. Van de Wiel, B.J.H., Ronda, R.J., Moene, A.F., De Bruin, H.A.R., Holtslag, A.A.M.: Intermittent turbulence and oscillations in the stable boundary layer. Part II: A system dynamics approach. J. Atmos. Sci. 59, 2567-2581 (2002b)

41. Van de Wiel, B.J.H., Moene, A.F., Hartogensis, O.K., De Bruin, H.A.R., Holtslag, A.A.M.: Intermittent turbulence and oscillations in the stable boundary layer. Part III: A Classification for observations during CASES99. J. Atmos. Sci. 60, 2509-2522 (2003)

42. Wyngaard, J.C.: On surface-layer turbulence. In: Haugen, D.A. (ed.) Workshop on Micrometeorology, pp. 101-149. Amer. Meteor. Soc., Boston (1973) 